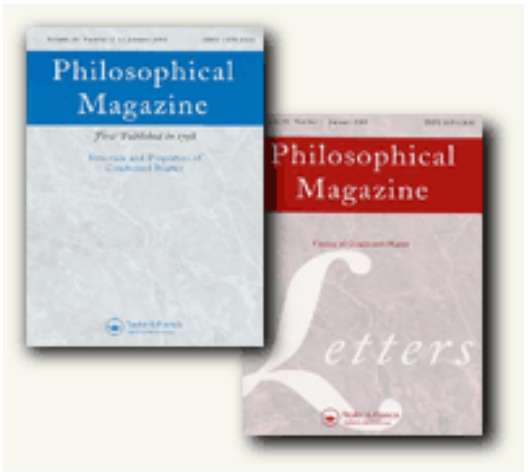

\title{
Kinetics versus thermodynamics in materials modelling: the case of the di-vacancy in iron
}

\begin{tabular}{|c|c|}
\hline Journal: & Philosophical Magazine \& Philosophical Magazine Letters \\
\hline Manuscript ID: & TPHL-09-Mar-0034.R2 \\
\hline Journal Selection: & Philosophical Magazine \\
\hline $\begin{array}{r}\text { Date Submitted by the } \\
\text { Author: }\end{array}$ & 23-Dec-2009 \\
\hline Complete List of Authors: & $\begin{array}{l}\text { Djurabekova, Flyura; University of Helsinki, Helsinki Institute of } \\
\text { Physics and Department of Physics; SCK-CEN, Structural Materials, } \\
\text { Institute of Nuclear Materials Science } \\
\text { Malerba, Lorenzo; SCK-CEN, Structural Materials, Institute of } \\
\text { Nuclear Materials Science } \\
\text { Pasianot, Roberto; CAC/CNEA, Deparamento de Materiales; } \\
\text { CONICET } \\
\text { Olsson, Pär; EDF-R\&D, Department MMC } \\
\text { Nordlund, Kai; University of Helsinki, Helsinki Institute of Physics } \\
\text { and Department of Physics }\end{array}$ \\
\hline Keywords: & diffusion, molecular dynamic simulations, Monte-Carlo, vacancies \\
\hline Keywords (user supplied): & \\
\hline
\end{tabular}

\section{s) ScholarONE" \\ Manuscript Central}




\section{Philosonhical}

Philosophical Magazine Letters

Vol._00MAPerbaa

${ }^{a}$ Structural Materials, Institute of Nuclear Materials Science, SCK•CEN, Boeretang 200, B-2400, Mol, Belgium; ${ }^{\mathrm{b}}$ Helsinki Institute of Physics and Department of Physics, P.O. Box 43,FIN-00014 University of Helsinki, Finland; ${ }^{\mathrm{c}}$ Depto. Materiales, CAC-CNEA, Avda. Gral. Paz 1499, 1650 San Martín, Pcia. Buenos Aires, Argentina; ${ }^{\mathrm{d}}$ CONICET, Avda. Rivadavia 1917, 1033 Buenos Aires, Argentina; e Dept. MMC, EDF-RED, Les Renardiéres, 77818 Moret-sur-Loing, France 


\title{
RESEARCH ARTICLE
}

\section{Kinetics versus thermodynamics in materials modelling: the case of the di-vacancy in iron}

(v2.0 released January 2008)

\begin{abstract}
Monte Carlo models are widely used for the study of microstructural and microchemical evolution of materials under irradiation. However, they often link explicitly the relevant activation energies to the energy difference between local equilibrium states. We provide a simple example (di-vacancy migration in iron) in which a rigorous activation energy calculation, by means of both empirical interatomic potentials and density functional theory methods, clearly shows that such a link is not granted, revealing a migration mechanism that a thermodynamicslinked activation energy model cannot predict. Such a mechanism is, however, fully consistent with thermodynamics. This example emphasises the importance of basing Monte Carlo methods on models where the activation energies are rigorously calculated, rather than deduced from widespread heuristic equations.
\end{abstract}

Keywords: Kinetic Monte Carlo, di-vacancy, vacancy cluster, diffusion, metals

\section{Introduction}

The microstructure and microchemical evolution in materials subjected to irradiation depends largely on the kinetics of thermally activated processes occurring at the atomic scale. Kinetic Monte Carlo (KMC) numerical methods are especially suitable to model the evolution of complex systems, when a large number of such processes are possible [?, ]]Chatt07,Fich91. They are therefore nowadays widely used, especially to treat problems of phase separation or damage evolution in materials subjected to annealing or irradiation [?, ]]Bouar02,Fu05,Krasn07,Domain99,Schmau02,Vincent08,Sois08. However, they require the precise knowledge of all involved activation energies, for all possible processes. The correct, on-the-fly evaluation of these energies, in such a way that the computational model is accurate, while remaining applicable in practice, is far from being a trivial problem. Even in the long studied case of atomic species redistribution in alloys via the vacancy diffusion mechanism [?, ]]Bouar02, Domain99, Schmau02,Vincent08, Young66 the energy barrier not only depends on the type of atom, but is also a complicated and a priori unknown function of the local atomic configuration [?, ]]Djurabek07a. Many heuristic approaches based on total energy calculations have been traditionally used [?, ]]Young66, Kang89 to allow at least partly for this dependence. The total energy of the system is calculated using either an empirical interatomic potential (EIP) [?, ]]Domain99, or pair interaction energies fitted either to an EIP [?, ]]Schmau02, Bouar02 or to density functional theory (DFT) calculations [?, ]]Vincent08, Sois08, often using a broken bond scheme [?, 1]Schmau02,Bouar02,Sois08. The main concern of these approaches is to ensure that the transition rates respect the detailed balance [?, ]lFich91, so as to be thermodynamically consistent. Often, for these conditions to be fulfilled, the characteristic energies of the relevant thermally-activated processes are explicitly correlated to energy differences between initial and final 


\section{nuary 22,2010
Page 3 of 12}

local equilibrium states [?, ]]Young66, Domain99,Vincent08.

However, the fact that a certain state is thermodynamically favoured does not necessarily mean that it is going to appear faster or more often than less favoured ones. The broken bond scheme, in which the activation energy is obtained as the difference between saddle point and initial energies, both expressed in terms of pair interactions [?, ]]Schmau02, Bouar02, Vincent08, Sois08, does attempt to be more realistic. Nonetheless, being based on a rigid lattice pair energy scheme, it remains a strongly simplified approach [?, ]]Djurabek07a.

One way of improving the accuracy of an atomistic KMC (AKMC) model in the sense discussed here is to feed it with activation energies rigorously calculated as functions of the local atomic configuration using, for example, an EIP, or DFT methods. If the number of involved energies is limited, this can be done by pre-tabulating all possibilities [?, ]]Djurabek07b, Barash05; if it is combinatorially large, advanced regression methods with good predictive capability (e.g. artificial intelligence) may be used [?, ]]Djurabek07a,Shastry05,Castin08c. In models of this type, thermodynamics does not appear explicitly in terms of binding energies, energy differences between states or favoured kinetic paths towards lower energy states. It is, instead, "hidden" in the difference between activation energies of direct and inverse processes, i.e., the energy difference between an initial state A and a final state $\mathrm{B}$ can be obtained as difference between the barrier from A to B and the barrier from $\mathrm{B}$ to $\mathrm{A}$.

In this Letter we present an application of such an AKMC model for the apparently simple case of the migration of a di-vacancy in iron to illustrate the fact that energy barriers dont always correlate with energy differences between initial and final state, as previously pointed out for the simpler case of a monovacancy [?, ]]Djurabek07a,Bocquet02. We choose to study this particular case since di-vacancies in iron (and probably in all or most bcc metals) are most stable in 2nn configuration, as consequence positron annihilation does not allow di-vacancies to be distinguished from single vacancies [?, ]]Kuri06. Thus, there is a lack of experimental data on di-vacancies in iron and this is in itself a motivation for our work. At the same time, iron is a material of primary importance for applications, within and without the nuclear industry, which is why a number a of EIPs to be compared exist. We show that the activation energies do not necessarily correlate with the energy difference between the involved local equilibrium states and may increase the frequency of appearance of states that are not necessarily the thermodynamically most favoured ones. Nonetheless, the model does respect thermodynamics, as is demonstrated by solving the corresponding master equations, which can be explicitly shown to embody Boltzmann's statistics (its stationary solution is thermodynamic equilibrium) [?, ]]VanKampen03, and comparing it to the AKMC simulation results. We also show, however, that even in a DFT framework relatively small differences between activation energies may appear, depending on the used approximation, with consequences that may be non-negligible a priori. In particular, our study reveals that the migration mechanism for the di-vacancy in iron is more complex than one would intuitively deduce from simple di-vacancy stability considerations. Such a mechanism cannot be predicted by a model that explicitly couples activation energies and energy differences between local equilibrium states and requires an atomic-level study to be clearly identified. 
Submitted for publication

\section{Methodology}

KMC methods determine the evolution of a system by stochastically choosing events whose probabilities are given in terms of frequencies. The latter are written using the classical transition-state-theory expression for the frequency of thermally activated phenomena: $\Gamma=\nu e^{-E_{a} / k_{B} T}$, where $k_{B}$ is Boltzmann's constant, T the absolute temperature, $\nu$ the attempt frequency (here considered constant and equal to $6 \times 10^{12} s^{-1}$ ), and $E_{a}$ the activation energy. The associated time is estimated using the residence time algorithm [?, ]]Bortz75. In our case, $E_{a}$ corresponds to the energy barrier for the exchange of a vacancy with a neighbouring atom. The possible states of the cluster were defined by the mutual distance between the two vacancies, in terms of nearest neighbour $(n n)$ shells. There are five possible states of the bound configurations of a di-vacancy: $1 n n, 2 n n, 3 n n, 4 n n$, and $5 n n$. The possible states and transitions are pictorially represented in Fig. 1. Three transitions, namely $3 n n \rightarrow \infty, 4 n n \rightarrow \infty$, and $5 n n \rightarrow \infty$, lead to di-vacancy splitting. The energy barriers for all possible transitions were calculated by the drag method [?, ]]Henkel00 using (for a check of trends) four widely used EIPs, denoted here as: AB [?, ]]Ackland97, MH [?, ]]Mend03, AM [?, ]]Ackland04, and DD [?, ]]Dudarev05. For these simple transitions, the drag method has been checked to provide the same results as the nudged-elastic band method (NEB) [?, ]] NEBb. All the classical potential calculations were carried out with the DYMOKA code [?, ]]DYMOKA. The corresponding DFT calculations were performed in two different ways, namely using pseudopotentials generated both within the projector augmented wave (PAW) approach [?, ]]Kresse99 and in the form of ultrasoft pseudopotentials (USPP) [?, ]]Kresse99, Vander90,Kresse96a, as implemented in the Vienna ab initio simulation package (VASP) [?, ]]Kresse96b. The USPP formalism can be derived from the PAW formalism by linearization. In general, the USPP method does not describe transition metals and magnetic systems as well as the PAW method does. However, a large amount of DFT data found in the literature concerning $\mathrm{Fe}$, and impurities in it, have been in fact obtained in the USPP approximation[?, ]]Domain01,Becquart03, Vincent05,Vincent06b and used to parametrise AKMC models $[?]$,$] Vincent08. All calculations were spin polarized and the exchange correlation$ functional used was the generalised gradient approximation [?, ]]PW91 with the Vosko-Wilk-Nusair correlation interpolation [?, ]]VWN for the PAW approach. The barriers were calculated using the nudged elastic band method [?, ]]NEBb implemented in VASP on supercells of 128 bcc sites at constant volume conditions. The reciprocal space was sampled with $27 \mathrm{k}$-points according to the method by [?, ]]MonkPack. The plane wave energy cutoff was $300 \mathrm{eV}$ for PAW and $240 \mathrm{eV}$ for USPP. The convergence using these parameters has been reported earlier [?, ]]Domain01, Olsson.

\section{Results and Discussion}

Fig. 2 shows the schematic energy landscape, according to the AKMC model, of di-vacancy states and transitions along the path connecting two dissociated states through, in order, $5 n n, 1 n n, 2 n n$, and $4 n n$ states. The dissociated state is the reference, i.e. has zero energy. According to all used model Hamiltonians, there are three bound states, namely (in order of increasing binding) $4 n n$, 1nn, and $2 n n$. DFT predicts also the $5 n n$ state to be bound (no EIP grasps this feature). According to USPP calculations $1 n n$ and $4 n n$ states are almost equal in energy, while all EIPs and PAW calculations predict $1 n n$ to be more strongly bound. Yet, all EIPs favour the transition from the $2 n n$ ground-state to $4 n n$ over 
1

4

5

7

8

9

10

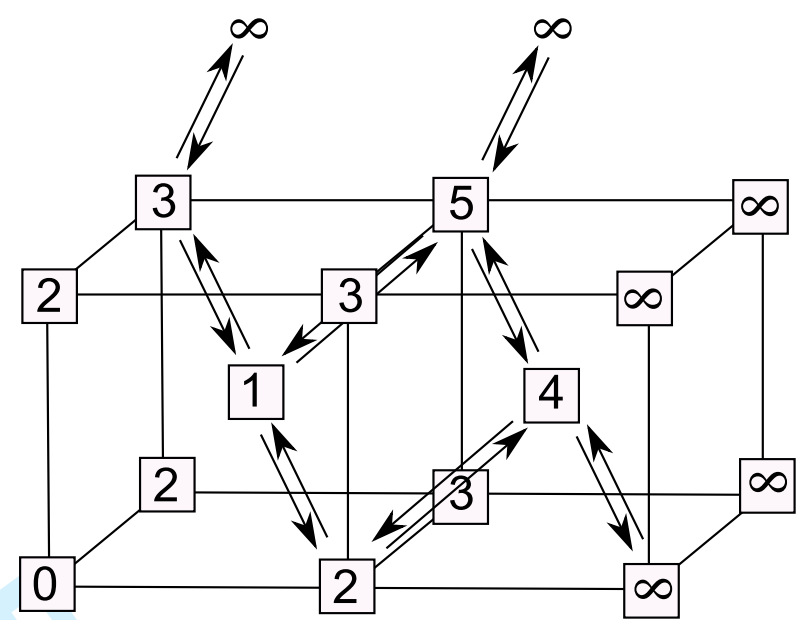

Figure 1. Different possible di-vacancy states and transitions. 0 is the position of the first vacancy; different states are obtained depending on the location of the second one. $\infty$ positions correspond to a dissociated di-vacancy. Transitions are possible only via the exchange of the given vacancy with a nearest neighbour atom (e.g. $1 \rightarrow 2$ ).

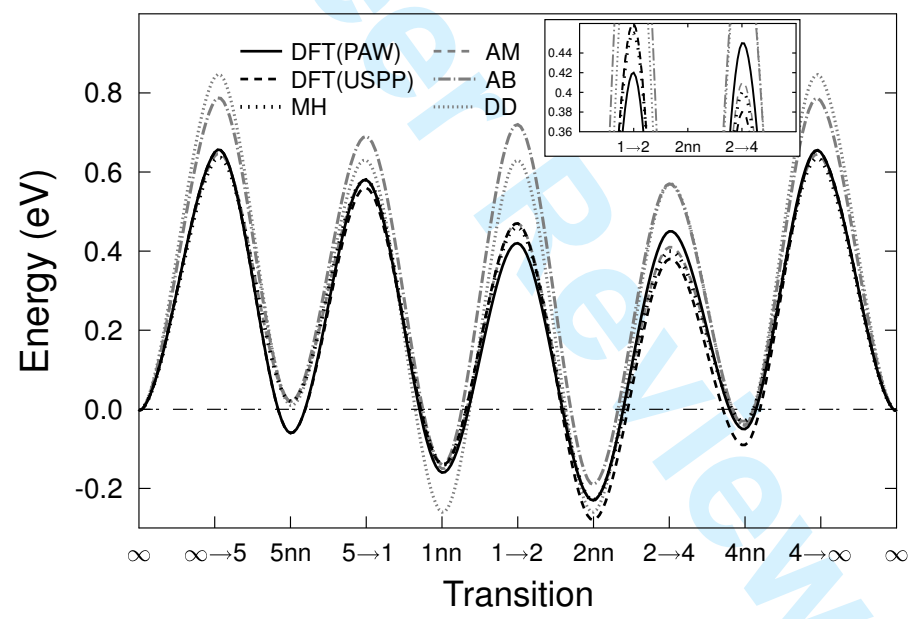

Figure 2. Schematic energy landscape (as implemented in the MC model) of di-vacancy states and transitions along an ideal path between two dissociated states (zero energy), according to different EIPs and DFT approximations. The bound states have negative energies.

the transition to $1 \mathrm{nn}$. Interestingly, the same result, though unnoticed, was also obtained with a pair potential for iron in a work published almost 40 years ago [?, ]]Tsai70. We also obtained the same trend using two other EIPs for Fe, from Ref. $[?$,$] Simon93 and from Ref.[?, ]]Chak02. Finally, this result is also provided by$ DFT(USPP) calculations. Conversely, according to DFT(PAW) both transitions are almost equally probable. Thus, all tested EIPs favour a transition to a less strongly bound, and therefore thermodynamically less favoured, state. In DFT the situation is smoothed: with USPP two energetically equivalent states are reached with different frequency, while with PAW two states of different stability are reached with essentially the same frequency. Regardless of the method differences, most important for the conclusions of the current work is that in no case is the energy barrier correlated with the energy difference between states, in agreement 
Submitted for publication

\begin{tabular}{|c|c|c|c|c|}
\hline EIP & $\begin{array}{c}E_{m} \\
(\mathrm{eV})\end{array}$ & $\begin{array}{c}D_{0} \times 10^{-3} \\
\left(\mathrm{~cm}^{2} / \mathrm{s}\right)\end{array}$ & $\begin{array}{c}E_{d} \\
(\mathrm{eV})\end{array}$ & $\begin{array}{c}\tau_{0} \times 10^{-15} \\
(\mathrm{~s})\end{array}$ \\
\hline PAW & $0.66 \pm 0.06$ & $3.6 \pm 0.74$ & $0.81 \pm 0.07$ & $9.16 \pm 2.2$ \\
\hline USSP & $0.68 \pm 0.09$ & $3.8 \pm 1.0$ & $0.82 \pm 0.09$ & $11.5 \pm 3.4$ \\
\hline AB [?, ]]Ackland97 & $0.75 \pm 0.08$ & $1.54 \pm 0.45$ & $0.92 \pm 0.1$ & $7.15 \pm 2.7$ \\
\hline DD [?, ]]Dudarev05 & $0.84 \pm 0.07$ & $2.09 \pm 0.56$ & $1.1 \pm 0.09$ & $9.45 \pm 3.6$ \\
\hline AM[?, ]]Ackland04 & $0.63 \pm 0.06$ & $2.9 \pm 0.6$ & $0.82 \pm 0.07$ & $7.71 \pm 1.8$ \\
\hline AM,eq.(1) & $0.64 \pm 0.08$ & $1.6 \pm 0.5$ & $0.83 \pm 0.07$ & $14 \pm 3.4$ \\
\hline
\end{tabular}

with [?, ]]Djurabek07a,Bocquet02. Of all EIPs, the one globally providing the closest results to DFT is AM (or, almost indifferently, MH) and was therefore used for the comparative studies.

The AKMC simulations were performed with pre-tabulated energy barrier values [?, ]]Djurabek07b, calculated as described above. The di-vacancy was always introduced in the simulation box in $2 n n$ state, which is the most stable according to all used model Hamiltonians. The cluster was considered to exist so long as the two vacancies did not part beyond $5 n n$ distance. The simulation was repeated 50 times at different temperatures between $250 \mathrm{~K}$ and $500 \mathrm{~K}$. The migration properties of the di-vacancy were studied in detail, by monitoring the visited configurations, their frequency, the time spent in each of them and the total lifetime of the complex, as well as its mean free path before dissociation. The effective migration and dissociation energies of the di-vacancy were obtained as slopes of the corresponding Arrhenius plots for the diffusion coefficients and lifetimes calculated with barriers from different EIPs, as well as from USPP and PAW DFT calculations (Table 1). In addition, for comparison the same type of simulation was also performed by estimating the energy barriers with the heuristic equation proposed in [?, ]]Domain99 and used, e.g., in [?, ]]Kang89:

$$
E_{b}=E_{0}+\frac{E_{f}-E_{i}}{2}
$$

where $E_{i}$ and $E_{f}$ are, respectively, the total energy before and after the vacancy jump, calculated on rigid lattice at each AKMC step using the AM potential. $E_{0}$ is a constant barrier, customarily assumed to equal the single vacancy migration energy in bcc-iron. The latter was calculated to be $0.63 \mathrm{eV}$ with the drag method using the $\mathrm{AM}$ and $\mathrm{MH}$ potentials. The barriers calculated from the different EIPs and DFT varieties provide different mean free paths of the di-vacancy, consistently with the different dissociation and migration energies predicted. Despite the fact that the DD EIP gives the highest effective migration energy for the di-vacancy, the even higher barrier for dissociation from the $4 n n$ state (Fig. 2) allows the di-vacancy to ramble the longest distance during its lifetime (Fig. 3).

Fig. 4 shows the occupation statistics of di-vacancy states $N_{i} /\left(N_{2}\left(n_{A K M C}=0\right)\right)$ versus AKMC steps according to AM-calculated barriers (Fig. 4(a)) and estimated 


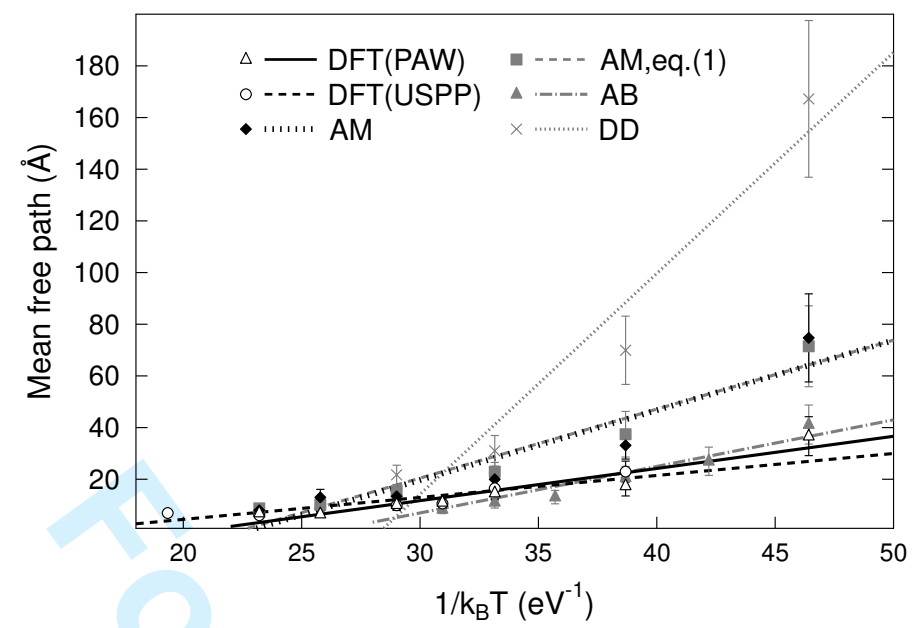

Figure 3. Mean free paths of di-vacancy against inverse temperature. The lines are guides to the eye.

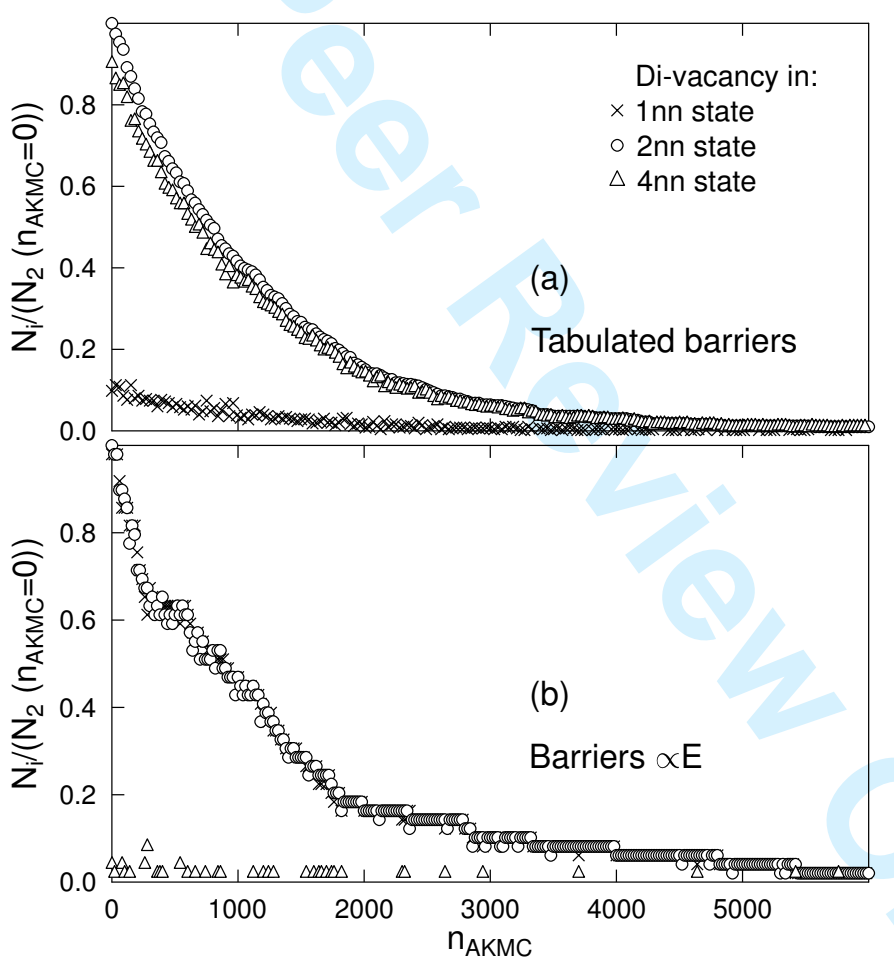

Figure 4. Occupation statistics of three bound di-vacancy states (1nn, 2nn, and 4nn) visited by one di-vacancy at each AKMC step during its diffusion migration. The di-vacancy always starts from $2 \mathrm{nn}$ state. The statistics was defined by 50 simulations at $300 \mathrm{~K}$ using (a) pre-tabulated barriers and (b) barriers from eq.(1), both with the same potential.

from eq. (1) (Fig. 4(b)), obtained as average over 50 different simulations at $300 \mathrm{~K}$. Here $N_{i}$ is the number of simulations in which the di-vacancy was found in the inn state at each AKMC step, $n_{A K M C}$, and $N_{2}\left(n_{A K M C}=0\right)=50$ the total number of simulations (every di-vacancy starts from the $2 n n$ state). The curves tend to zero due to the eventual dissociation of all di-vacancies. Consistently with the energy landscape of Fig. $2,2 n n$ and $4 n n$ states are by far the most often observed ones. 
This means that the di-vacancy migrates by oscillating between $2 n n$ and $4 n n$ states rather than $2 n n$ and $1 n n$ (Fig. 4(a)). What would be intuitively expected, knowing that the $1 n n$ state is more strongly bound than $4 n n$ is that, on the contrary, while migrating the di-vacancy should oscillate between $2 n n$ and $1 n n$ states. The latter mechanism, which is the only one that has been considered even in recent DFT investigations [?, ]]Fu05, is, not surprisingly, predicted by an AKMC model in which eq. (1) is used (Fig. 4(b)). Here the presence of $2 n n$ and $1 n n$ states is very clear while the $4 n n$ state hardly ever appears.

A different migration mechanism should imply, in principle, also different effective migration and dissociation energies and, therefore, a different effect on mass transportation in metal alloys of interest due to the thermal diffusion. Although we do observe differences between the diffusion parameters assessed from AKMC simulations with tabulated barriers and with barriers obtained from eq. (1), this difference is surprisingly small. The involved energy values are rather similar as there is an accidental compensation between predicted migration energies and binding energies in rigid lattice (eq. (1)) with the AM potential. However, we emphasise that in different circumstances (no rigid lattice, different EIP) this similarity will most likely vanish, and hence it is important to know the true mechanism of cluster migration.

The observed counterintuitive divacancy migration mechanism is a direct consequence of the values of the energy barriers. The question to be answered is whether the $2 n n-4 n n$ mechanism is consistent with thermodynamics, because Fig. 4(a) may wrongly lead to the conclusion that Boltzmann's statistics is violated, if a higher energy state is occupied much more often than a lower energy state. To answer this question it is necessary to trace the probability density of states as a function of time, instead of AKMC steps, since the residence time in the different states is different, regardless of how often they appear. This is obtained in a natural way by solving the master equation system that governs the process [?, ]]Fich91, Chatt07. Note that we are solving here the master equation for an open system, consistent with the AKMC simulations (where we start the simulations from a di-vacancy and we stop collecting data when the di-vacancy dissociates):

$$
\left\{\begin{aligned}
\frac{d C_{1}}{d t}= & K^{21} \cdot C_{2}+K^{31} \cdot C_{3}+K^{51} \cdot C_{5} \\
& -\left(K^{12}+K^{13}+K^{15}\right) \cdot C_{1} \\
\frac{d C_{2}}{d t}= & K^{12} \cdot C_{1}+K^{42} \cdot C_{4}-\left(K^{21}+K^{24}\right) \cdot C_{2} \\
\frac{d C_{3}}{d t}= & K^{13} \cdot C_{1}+K^{43} \cdot C_{4}-\left(K^{31}+K^{34}+K^{3 \infty}\right) \cdot C_{3} \\
\frac{d C_{4}}{d t}= & K^{24} \cdot C_{2}+K^{34} \cdot C_{3}+K^{54} \cdot C_{5} \\
& -\left(K^{42}+K^{43}+K^{45}+K^{4 \infty}\right) \cdot C_{4} \\
\frac{d C_{5}}{d t}= & K^{15} \cdot C_{1}+K^{45} \cdot C_{4}-\left(K^{51}+K^{54}+K^{5 \infty}\right) \cdot C_{5}
\end{aligned}\right.
$$

Here $C_{i}(i=1,2, \ldots, 5)$ denote the probability density of di-vacancies in the $i n n$ state at a given time. The solution of the equations was obtained with the initial condition: $C_{2}=1$ and $C_{1}=C_{3}=C_{4}=C_{5}=0$ at $300 \mathrm{~K}$. The $K^{i j}$ coefficients are defined as: 


\section{nuary 22,2010
Page $\mathbf{9}$ of $\mathbf{1 2}$ \\ Philosophical Magazine \& Philosophical Magazine Letters}

Philosophical Magazine Letters

$K^{i j}=k^{i j} \cdot \Gamma^{i j}=k^{i j} \cdot \nu_{0} e^{-E_{m}^{i j} / k_{B} T}$

i.e. they are obtained by multiplying the rate $\Gamma^{i j}$ of the $i \rightarrow j$ transition, times the number of possible ways in which this transition may occur, $k^{i j}$ (multiplicity of equivalent transitions). These multiplicities solely depend on crystallographic structure and geometry of the cluster: $k^{12}=k^{13}=k^{54}=6 ; k^{15}=k^{42}=k^{45}=k^{51}=2$; $k^{21}=k^{24}=k^{34}=k^{4 \infty}=k^{5 \infty}=8 ; k^{31}=k^{3 \infty}=k^{43}=4$. All the multiplicities are doubled taking into account the equal probabilities of both vacancies to jump for each transition. Multiplicities of not listed transitions $k^{i j}$ are zero as they are impossible in the bcc-structure.

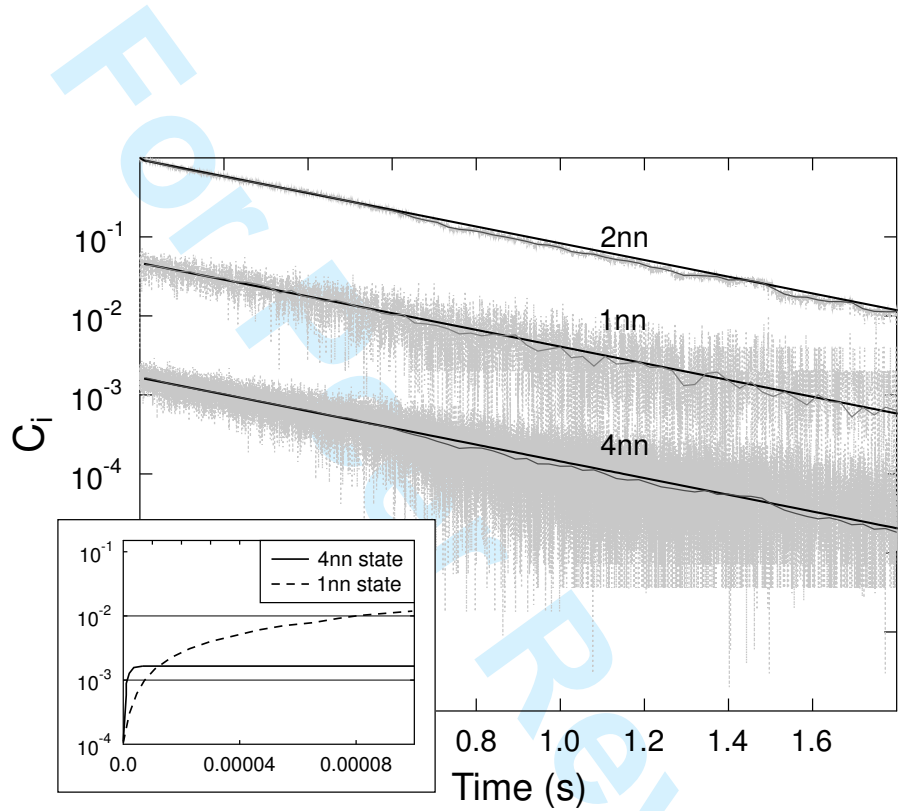

Figure 5. Probability density of di-vacancy states $C_{i}=N_{i} /\left(N_{2}(t=0)\right)$ at $300 \mathrm{~K}$ as a function of time for the same bound states as in Fig. 4, using barriers from the AM potential. $N_{i}$ is the number of simulations in which the di-vacancy is found in the inn state within the considered $\Delta t<10^{-5} ; N_{2}(t=0)$ $=500$ is the total amount of the simulations, each starting from a di-vacancy in $2 n n$ state. The dark grey lines are "horizontal" averages of the light grey ones over larger time intervals $\left(\Delta t \sim 3 \times 10^{-3} \mathrm{~s}\right)$. The solid black lines are the solutions of eqs. (2). The inset (left bottom corner) zooms in the beginning of the $1 n n$ and $4 n n$ distributions.

In Fig. 5 we compare the results for the probability density of states corresponding to the AKMC simulations of Fig. 4(a), plotted versus time, with the numerical solution of eqs. (2) at $300 \mathrm{~K}$ (solid black lines). We increased the statistics to 500 AKMC simulations to reduce the statistical fluctuations. The comparison requires a proper treatment of the AKMC simulation data, because time advances at a different pace in each simulation according to residence time algorithm. This happens because the $\Delta t$ associated with each AKMC step is not constant, thus the same number of AKMC steps corresponds to a different time. When the statistical averaging is applied (as described in the figure caption), the AKMC results are directly comparable, and in fact in excellent agreement, with the solution of the master equations (2).

The probability that, in an infinitesimal time interval, the di-vacancy is found in a $2 n n$ state is always the highest, followed by $1 n n$ (about one order of magnitude lower) and, finally, $4 n n$ (about two orders of magnitude lower). This result is fully consistent with the binding energies of Fig. 2, i.e. with the thermodynamics 
embodied by the EIP, even though the migration mechanism favours the appearance of the $4 \mathrm{nn}$ state, as shown in Fig. 4(a). Analytically, under a few simplifying assumptions, it is possible to show that the solution of the master equations corresponds indeed to classical Boltzmann's statistics. For example, if we do not allow dissociation in eqs. (2), the ratio between the concentrations of $1 n n$ and $4 n n$ divacancies after infinite time (obtained imposing that the derivatives on the left hand are zero), $C_{4}^{\infty} / C_{1}^{\infty}$, after neglecting $C_{3}^{\infty}$ and $C_{5}^{\infty}$ (which have been found to be very small) is:

$$
\frac{C_{1}^{\infty}}{C_{4}^{\infty}} \approx \frac{K^{42} K^{21}}{K^{12} K^{24}} \propto \exp \left[\frac{\left(E_{b}^{1}-E_{b}^{4}\right)}{k_{B} T}\right]
$$

where we have equated $E_{m}^{24}-E_{m}^{42}=E_{b}^{2}-E_{b}^{4}$ and $E_{m}^{21}-E_{m}^{12}=E_{b}^{2}-E_{b}^{1}$, the binding energies $E_{b}^{i}$ being positive values (depth of the wells in Fig. 2). This ratio, using AM binding energies, will be significantly larger than 1 for all temperatures of interest. On the other hand, it is clear also from Fig. 5 that the $C_{1} / C_{4}$ ratio remains essentially constant, on average, at a value much larger than 1 (about 28, as has been verified), independently of the fact that the total di-vacancy population is dwindling. This means that the steady-state in terms of visited configurations and also time spent in them is reached almost instantaneously and that, correspondingly, the time spent in the $1 n n$ state is always larger than in $4 n n$. Nonetheless, so long as the barriers are those predicted by the model Hamiltonian we have used, the mechanism of migration of the di-vacancy remains $2 n n-4 n n$, i.e. the number of times the $4 n n$ configuration is visited is significantly larger than for $1 n n$, even though the time spent in the former is shorter. This is implicitly demonstrated by the inset in the lower left corner of Fig. 5, which blows up the beginning of the curves. Since at the outset all di-vacancies are in the $2 n n$ state, both $1 n n$ and $4 n n$ curves start from zero but, of the two, the $4 n n$ one grows faster and reaches earlier the steady-state (before di-vacancies start to dissociate), in agreement with the faster kinetics dictated by the AM energy barriers of Fig. 2.

We also performed AKMC simulations with the barriers that should be considered the most reliable ones, i.e. those obtained from DFT(PAW) calculations. In this case, the migration mechanism was somewhat different, namely a mixture of $2 n n-1 n n$ and $2 n n-4 n n$ exchanges.

\section{Conclusions}

In summary, we have shown how a kinetic path can be "thermodynamically counterintuitive", as a consequence of the relative value of the involved activation energies, while in fact fully respecting the laws of thermodynamics. If a proper calculation of all relevant activation energies is performed, the mechanism of migration of the di-vacancy in iron consists in an oscillation between not only $2 n n$ and $1 n n$ states, but also, largely, between $2 n n$ and $4 n n$ states, despite the fact that the $1 n n$ state is the closest in energy to the fundamental $2 n n$ state. Such a "thermodynamically counterintuitive" mechanism could not be predicted by a model linking explicitly the activation energies to the energy difference between initial and final state. The use of models in which the kinetics is explicitly linked to 
these energy differences [?, ]] Young66, Domain99,Vincent08 may therefore suggest mechanisms that, though thermodynamically acceptable, do not in fact occur. This emphasises the importance of devoting great care and effort to a proper evaluation of activation energies, to ensure the accuracy of models describing the evolution of physical systems driven by thermally activated processes, possibly avoiding to resort to traditionally used heuristic equations.

This work was performed in the framework of the bilateral collaboration agreement between SCK•CEN and CNEA, sponsored by the Belgian Scientific Policy Office, under contract BL/52/A01. The DFT calculations were performed on the supercomputers at Centre de Calcul Recherche et Technologie (CCRT) in the framework of an EDF-CEA contract.

\section{References}

42

1 A. Chatterjee and D. Vlachos, ..., J. Computer-Aided Mater. Des 14 (2007), p. 253.

2 K.A. Fichthorn and W.H. Weinberg, ..., J. Chem. Phys. 95 (1991).

3 Y. Le Bouar and F. Soisson, ..., Phys. Rev. B 65 (2002), p. 094103.

4 C.C. Fu et al., ..., Nature Mater. 4 (2005), p. 68.

5 P. Krasnochtchekov, R.S. Averback, and P. Bellon, ..., Phys. Rev. B 75 (20078), p. 144107.

6 C. Domain, C.S. Becquart, and J.C.V. Duysen, ..., Mater. Res. Soc. Symp. Proc. 540 (1999), p. 643.

7 S. Schmauder and P. Binkele, ..., Comp. Mater. Sci. 25 (2002), p. 174.

8 E. Vincent et al., Precipitation of the FeCu system: A critical review of atomic kinetic Monte Carlo simulations, J. Nucl. Mater. 373 (2008), p. 387.

9 F. Soisson and C.C. Fu, ..., Phys. Rev. B 76 (2007), p. 214102.

10 W.M. Young and E.W. Elcock, ..., Proc. Phys. Soc. 89 (1966), p. 735.

11 F.G. Djurabekova et al., Artificial intelligence applied to atomistic kinetic Monte Carlo simulations in Fe-Cu alloys, Nucl. Inst. \& Meth. in Phys. Res. B 255 (2007), p. 8.

12 H.C. Kang and W.H. Weinberg, ..., J. Chem. Phys. 90 (1989), p. 2824.

13 F.G. Djurabekova et al., Stability and mobility of small vacancy and copper-vacancy clusters in bcc-Fe: An atomistic Kinetic Monte Carlo study, Nucl. Inst. \& Meth. in Phys. Res. B 255 (2007), p. 45 .

14 A.V. Barashev, ..., Philos. Mag. 85 (2005), p. 1539.

15 K. Shastry et al., ..., Phys. Rev. B 72 (2005), p. 085438.

16 N. Castin et al., Modelling radiation-induced phase changes in binary FeCu and ternary FeCuNi alloys using an artificial-intellegence-based atomistic kinetic Monte-Carlo approach, Nucl. Instr. \& Meth. in Phys. Res.B (2009) in press.

17 J.L. Bocquet, ..., Defect and Diffusion Forum 203-205 (2002), p. 81.

18 J. Kuriplach et al., Vacancy-solute complexes and their clusters in iron, APPLIED SURFACE SCIENCE 252(9) (2006), pp. 3303-3308.

19 N.G.V. Kampen, 2003, V. in Stochastic Processes in Physics and Chemistry North Holland.

20 A.B. Bortz, M.H. Kalos, and J.L. Lebowitz, .., J. Comp. Phys. 17 (1975), p. 10.

21 G. Henkelman, G. Johannesson, and H. Jonsson, 2000in Progress on Theoretical Chemistry and Physics Kluwer Academic, Dordrecht.

22 G.J. Ackland et al., ..., Phil. Mag. A 75 (1997), p. 713.

23 M. Mendelev et al., ..., Phil. Mag. 83 (2003), p. 3977.

24 G.J. Ackland et al., ..., J. Phys.: Condens. Matter 16 (2004), p. 1.

25 S.L. Dudarev and P.M. Derlet, ..., Phys.: Condens. Matter. 17 (2005), p. 7097 potential 2.

26 H. Jonsson, G. Mills, and K.W. Jacobsen, 1998, Nudged Elastic Band Method for Finding Minimum Energy Paths of Transitions. in Classical and Quantum Dynamics in Condensed Phase Simulations World Scientific.

27 C.S. Becquart et al., Massively parallel molecular dynamics simulations with EAM potentials, Radiat. Eff. Def. Solids 142 (1997), p. 9. 
28 G. Kresse and D. Joubert, Phys. Rev. B 59 (1999), p. 1758.

29 D. Vanderbilt, Phys. Rev. B 41 (1990), p. 7892.

30 G. Kresse and J. Hafner, J. Phys.: Condens. Matter 6 (1996), p. 8245.

31 G. Kresse and J. Furthmüller, Phys. Rev. B 54 (1996), p. 11169.

32 C. Domain and C.S. Becquart, Phys. Rev. B 65 (2001), p. 024103.

33 C.S. Becquart and C. Domain, Nucl. Instr. \& Meth. Phys. Res. B 202 (2003), p. 44.

34 E. Vincent, C.S. Becquart, and C. Domain, Nucl. Instr. \& Meth. Phys. Res. B 228 (2005), p. 137.

35 E. Vincent, C.S. Becquart, and C. Domain, J. Nucl. Mater. 359 (2006), p. 227.

36 J. Perdew et al., Atoms, molecules, solids, and surfaces: Applications of the generalized gradient approximation for exchange and correlation, Phys. Rev. B 46 (1992), p. 6671.

37 S.H. Vosko, L. Wilk, and M. Nusair, Can. J. Phys. 58 (1980), p. 1200.

38 H.J. Monkhorst and J.D. Pack, Phys. Rev. B 13 (1976), p. 588.

39 P. Olsson, C. Domain, and J. Wallenius, Phys. Rev. B 75 (2007), p. 014110.

40 D.H. Tsai, R. Bullough, and R.C. Perrin, ..., J. Phys. C: Solid St. Phys. 3 (1970), p. 2022.

41 G. Simonelli, R. Pasianot, and E.J. Savino, ..., Mat. Res. Soc. Symp. Proc. 291 (1993), p. 567 potential A.

42 R. Chakarova, V. Pontikis, and J. Wallenius, Development of $\mathrm{Fe}(\mathrm{bcc})$ - $\mathrm{Cr}$ many body potential and cohesion model, , WP6 Delivery report Nr.6, SPIRE project, EC contract no. FIKW-CT2000-00058, 2002 Available from:〈www.neutron.kth.se/publications/library/DR-6.pdf〉. 